\title{
Toward Population Management in an Integrated Care Model
}

\author{
Franklin W. Maddux ${ }^{\mathrm{a}}$ Stephen McMurray ${ }^{\mathrm{b}}$ Allen R. Nissenson ${ }^{\mathrm{b}}$ \\ ${ }^{a}$ Fresenius Medical Care, Waltham, Mass., and b DaVita, Inc., El Segundo, Calif., USA
}

\begin{abstract}
Under the Patient Protection and Affordable Care Act of 2010, accountable care organizations (ACOs) will be the primary mechanism for achieving the dual goals of high-quality patient care at managed per capita costs. To achieve these goals in the newly emerging health care environment, the nephrology community must plan for and direct integrated delivery and coordination of renal care, focusing on population management. Even though the ESRD patient population is a complex group with comorbid conditions that may confound integration of care, the nephrology community has unique experience providing integrated care through ACO-like programs. Specifically, the recent ESRD Management Demonstration Project sponsored by the Centers for Medicare \& Medicaid Services and the current ESRD Prospective Payment System with it Quality Incentive Program have demonstrated that integrated delivery of renal care can be accomplished in a manner that provides improved clinical outcomes with some financial margin of savings. Moving forward, integrated renal care will probably be linked to provider performance and quality outcomes measures, and clinical integration initiatives will share several common elements, namely performance-based payment models, coordination of communication via health care information technology, and development of best practices for care coordination and resource utilization. Integration initiatives must be designed to be measured and evaluated, and, con-
\end{abstract}

sistent with principles of continuous quality improvement, each initiative will provide for iterative improvements of the initiative.

(c) 2013 S. Karger AG, Basel

\section{Introduction}

The Patient Protection and Affordable Care Act of 2010 (PPACA) advanced the national health care reform discussion by extending health care insurance to almost all Americans while simultaneously emphasizing delivery of high-quality, patient-centered health care at lowerthan-current costs [1]. Although the constitutionality of the PPACA was challenged by 26 states in numerous federal courts, in June 2012 the Supreme Court of the United States upheld a vast majority of the legislation, ruling that the 'individual mandate,' which requires nearly all Americans to purchase health care insurance or pay a penalty for declining coverage, was constitutional under Congress's power to tax. The Court invalidated several PPACA provisions that would have compelled states to expand Medicaid programs [2].

Reproduced with permission of the Clinical Journal of the American Society of Nephrology and of the American Society of Nephrology via the Copyright Clearance Center.

\section{KARGER}

E-Mail karger@karger.com

www.karger.com/bpu
(C) 2013 S. Karger AG, Basel

0253-5068/13/0364-0152\$38.00/0
Dr. Allen R. Nissenson

DaVita, Inc.

601 Hawaii Street

El Segundo, CA 90245 (USA)

E-Mail Allen.Nissenson@ davita.com 
Under the PPACA, accountable care organizations (ACOs) will be the primary mechanism for aligning quality of care and low costs. An ACO is a providerinitiated and -led collaboration of caregivers who assume responsibility to deliver and manage the entire spectrum of care for a cohort of beneficiaries while reducing the cost of care for that same cohort. Therefore, ACOs are characterized by a collective accountability for quality and cost, payments that are linked to quality measures and quality improvements, and performance measures designed to assure that cost savings occur through improved quality [3]. To incentivize the dual goals of high-quality care and cost savings, an ACO is offered one of two payment options. The first option allows the ACO to receive a portion of cost savings with no penalty in the event of excess costs; the second option, however, shares a larger portion of cost savings but requires the ACO to repay a portion of any excess cost. The financial incentives of shared cost savings are linked to ACO quality scores in 33 predefined quality benchmarks that are grouped into four categories: patient/caregiver experience, care coordination/patient safety, preventative health, and at-risk population/frail elderly health.

For an ACO to achieve the quality performance measures that are tied to incented cost savings, a high level of collaboration, integration of care, and population management will necessarily be required of the primary, specialty, and allied health care providers within the ACO. In fact, economic modeling suggests that integrated delivery of care is sufficient to drive improved quality and reduced costs [4]. However, because patients with ESRD present with a unique clinical profile that requires specialized, high-cost interventions, it is yet unclear whether the ESRD population would be best served within a general ACO or within a renal-focused ACO-like integrated care program $[5,6]$. Throughout this discussion we refer to the renal ACO as one that would serve patients with ESRD. It is clear, however, that ideally a renal ACO would include patients with advanced CKD. Slowing the progression of CKD and appropriately managing the transition from CKD to ESRD are of fundamental importance to the health of this vulnerable population and essential to control overall costs of care. The adoption of coordination or care extending into the CKD population is difficult to institute but should be an important evolutionary step to the renal ACO model.

A recent 5-year demonstration project has tested renal-specific integrated delivery of care using an ACOlike model that used a fixed payment scheme under which providers assumed risk for the total cost of care. In the
ESRD Management Demonstration Project sponsored by the Centers for Medicare \& Medicaid Services, the effect of integrated care for an ESRD population, as managed by three large dialysis providers, was analyzed on the basis of clinical outcomes (e.g., hospitalizations, mortality, quality of life, patient satisfaction) and cost reductions [7, 8]. The results of the demonstration through the first 3 years were mixed, with positive trends in some but not all clinical areas (tables 1 and 2): Mortality and all-cause hospitalization rates decreased, diabetic care and immunizations increased, and patient satisfaction remained high. Cost reductions, however, were not demonstrated by the primary analysis after 3 years: Overall costs were reduced for one provider, whereas only hospital and inpatient costs were reduced for a second provider compared with matched fee-for-service patients $[9,10]$.

Although the ESRD Management Demonstration Project was an opportunity to test integrated renal care within an ACO-like model, the nephrology community continues to function under the Medicare ESRD Prospective Payment System (ESRD PPS), which was implemented on January 1, 2011, and resulted from the Medicare Improvements for Patients and Providers Act of 2008 [11]. Before this payment system, the Medicare ESRD system reimbursed providers via a composite payment that included dialysis-related service $s$ and limited feefor-service items. The new payment system, however, implemented a 'bundle' payment that issued a fixed per-dialysis-session payment, combining the previously separated composite and fee-for-service payments. The goal of this fixed-payment bundle system is to optimize utilization of renal-related medications while simultaneously incentivizing home dialysis and ESRD-related clinical laboratory tests, thereby creating a greater cost savings margin for the provider [11].

The financial risk and responsibility for integrated care were further shifted onto dialysis facilities when the ESRD Quality Incentive Program (QIP) was implemented on January 1, 2012. Established to define a basic level of care, the ESRD QIP is a performance-based payment system that measures patient outcomes against prespecified outcomes benchmarks. For providers not meeting performance criteria, the payment may be reduced by as much as $2 \%$ per year through the ESRD QIP. Taken together, the ESRD PPS and QIP create a system that is analogous to the ACO proposed by the PPACA and the ACO-like model tested in the ESRD Management Demonstration Project. Therefore, the nephrology community is uniquely situated to lead the efforts of health care reform and integration of disease-specific care delivery. 
Table 1. Effect of interventions in the ESRD Management Demonstration Project

\begin{tabular}{|c|c|c|}
\hline DMO A & DMO B & DMO C \\
\hline $\begin{array}{l}\text { Impact of Pharmacist Involvement on } \\
\text { Medication-Related Problems (Chapter 3) } \\
\text { - Increase in medication-related problems } \\
\text { over time } \\
\text { Management of CVD and Cardiovascular } \\
\text { Risk Factors (Chapter 4) } \\
\text { - Increase in ACEi/ARB use versus baseline } \\
\text { among patients with congestive heart } \\
\text { failure enrolled for one year, yet a } \\
\text { decrease to below baseline at } 2 \text { years } \\
\text { - No improvement in BP control among } \\
\text { all enrollees } \\
\text { Improving Preventive Care Processes } \\
\text { (Chapter 5) } \\
\text { - More patients received influenza and } \\
\text { pneumococcal vaccinations in } 2007 \text { and } \\
\text { 2008 than in FFS } \\
\text { - Significantly more patients with diabetes } \\
\text { mellitus received routine HbA1c tests in } \\
\text { accordance with established guidelines } \\
\text { compared with FFS and a nationally } \\
\text { representative population of HD patients } \\
\text { (US DOPPS) comparison populations } \\
\text { - More patients with diabetes mellitus } \\
\text { received routine foot and retinal } \\
\text { examinations by mid-2008 compared } \\
\text { with US DOPPS }\end{array}$ & $\begin{array}{l}\text { Improving Advanced Care Planning } \\
\text { (Chapter 6) } \\
\text { - Slight increase in adoption of ACP for } \\
\text { HD patients versus baseline }{ }^{\mathrm{a}} \\
\text { Improving Diabetes } \\
\text { Management (Chapter 7) } \\
\text { - Increase in HbA1c tests for patients } \\
\text { with diabetes mellitus during period of } \\
\text { standing orders } \\
\text { - Sharp decrease in HbA1c measurement } \\
\text { among patients with diabetes mellitus } \\
\text { after standing orders were discontinued } \\
\text { - No significant change in achievement of } \\
\text { the HbA1c target } \\
\text { (HbA1c }<7 \% \text { ) } \\
\text { Changing Prescription Patterns of ACEi/ } \\
\text { ARB Use (Chapter } 8 \text { ) } \\
\text { - Increase in ACEi/ARB use versus baseline } \\
\text { among patients with persistent } \\
\text { hypertension enrolled at least } 2 \text { years }\end{array}$ & $\begin{array}{l}\text { Use of Oral Nutritional Supplement in } \\
\text { Patients with Low Serum Albumin } \\
\text { (Chapter 9) } \\
\text { - Significantly reduced mortality among } \\
\text { patients with the clinical indication } \\
\text { to receive ONS as compared with the } \\
\text { CMS ESRD Clinical Performance } \\
\text { Project population } \\
\text { - Increase in serum albumin among } \\
\text { patients with the clinical indication } \\
\text { to receive ONS } \\
\text { Impact of Home Weight Monitoring on } \\
\text { Clinical Outcomes (Chapter 10) } \\
\text { - 42\% of all patients participated in the } \\
\text { HWM program; however, 70\% of } \\
\text { 2006 enrollees used HWM and only } \\
\text { 16\% of 2007/2008 enrollees ever used } \\
\text { HWM } \\
\text { - Short-term effect of reducing IDWG } \\
\text { for patients on HWM } \\
\text { - No sustained effect of HWM in DWG } \\
\text { after discontinuation } \\
\text { - HWM was associated with lower } \\
\text { all-cause and cardiovascular mortality } \\
\text { and all-cause and cardiovascular } \\
\text { hospitalization for } 2006 \text { enrollees but }\end{array}$ \\
\hline
\end{tabular}

$\mathrm{DMO}=$ Disease management organization; $\mathrm{ACP}=$ advanced care plans; $\mathrm{HD}=$ hemodialysis; ONS = oral nutritional supplements; CMS = Centers for Medicare \& Medicaid Services; CVD = cardiovascular disease; $\mathrm{ACEi}$ = angiotensin-converting enzyme inhibitor; $\mathrm{ARB}=$ an- giotensin receptor blocker; HbA1c $=$ hemoglobin A1c; HWM = home weight monitoring; IDWG = interdialytic weight gain; FFS = fee-for-service; CVD = cardiovascular disease; US DOPPS = US Dialysis Outcomes and Practice Patterns Study. Reprinted from reference 8.

a Analysis is limited by inconsistent ACP data, lack of an adequate comparison population, and the small number of patients included in the analyses.
Although the ESRD PPS and QIP approach taken by the Centers for Medicare \& Medicaid Services is a step toward the ACO model, an important element of the former approach is missing. First, the QIP to date focuses on dialysis-related intermediate outcomes, many of which may only marginally affect primary outcomes in patients with ESRD. In addition, the best facilities under this system would earn back only the full bundled payment that would have been received before the QIP implemented. The ACO approach, on the other hand, as will be described later, is based on a shared savings or full capitation approach. Under this ACO system, delivering the highest-quality care minimizes unnecessary hospitalizations, and the money saved as a result is available to the providers to further enhance the care delivery processes and reward providers for driving the best clinical outcomes.

Whether ESRD care is delivered through a general ACO, a renal-focused ACO, or the current ESRD PPS with its QIP, the nephrology community must consider and drive the integration and coordination of renal care delivery. Fundamentally, the shift toward integrated care requires a shift toward population management. To that end, the ACO will manage the population while individual providers within the ACO will manage individual patient care. Additionally, the ACO, whether general or renal-focused, has a responsibility to provide full-spectrum health care to the attributed population, including renal 
Table 2. Outcome measures during the ESRD Management Demonstration Project

\begin{tabular}{|c|c|c|}
\hline DMO A & DMO B & DMO C \\
\hline $\begin{array}{l}\text { - No significant survival advantage over } \\
\text { FFS at } 1 \text { and } 2 \text { years } \\
\text { - No significant difference in all-cause } \\
\text { and cardiovascular hospitalizations } \\
\text { compared with FFS at } 1 \text { and } 2 \text { years } \\
\text { - Hospital admission and readmission } \\
\text { rates were not significantly different } \\
\text { from FFS } \\
\text { - Significantly fewer SNF stays and } \\
\text { physician visits than FFS } \\
\text { - Significantly higher rates of transplant } \\
\text { wait-listing compared with FFS, but no } \\
\text { significant difference in transplantation } \\
\text { percentages compared with FFS }\end{array}$ & $\begin{array}{l}\text { - Significant survival advantage over FFS at } \\
1 \text { year and } 2 \text { years } \\
\text { - Significantly lower percentage of } \\
\text { cardiovascular hospitalizations but not } \\
\text { all-cause hospitalization compared with } \\
\text { FFS at } 2 \text { years (possibly an artifact of } \\
\text { - limited data) Hospital admission rates } \\
\text { were not significantly different from FFS; } \\
\text { readmission rates exceeded FFS } \\
\text { - Significantly fewer physician visits than } \\
\text { FFS } \\
\text { No significant difference in ED visits or } \\
\text { SNF stays compared with FFS over 3-year } \\
\text { evaluation period } \\
\text { - Transplantation rates were significantly } \\
\text { lower than FFS by year } 2 \text { and transplant } \\
\text { wait-listing rates were significantly lower } \\
\text { compared with FFS }\end{array}$ & 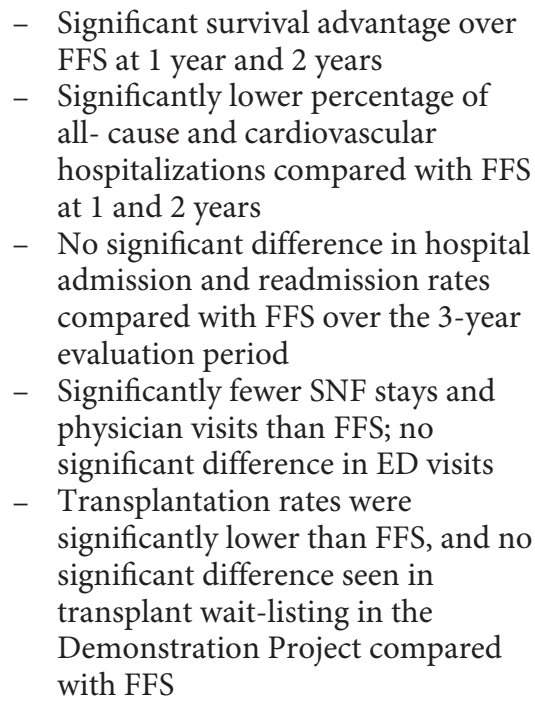 \\
\hline
\end{tabular}

Service utilization analyses were conducted with multiple methods; only results consistent across all methods are highlighted here. $\mathrm{DMO}=$ Disease management organization; FFS = fee-for-service; SNF = skilled nursing facility; ED = emergency department. Reprinted from reference 8 .

patients. To define and track such population management successes, renal care integration initiatives must be quantified and evaluated using appropriate clinical data to provide evidence-based delivery statistics, iterative improvements to the integration efforts, and population-focused treatment guidelines. Moving forward, delivery of integrated renal care will most certainly be tied to physician and dialysis provider performance and quality outcome benchmarks, as in the ESRD QIP and ESRD Management Demonstration Project. As a consequence, the nephrology community must prospectively recognize the broader clinical and financial issues of linking renal-related and non-ESRD costs. For example, outcomes measures may no longer be solely tied to dialysis and renal costs but may be additionally tied to such services as skilled nursing, nutrition and fluid management, or depression counseling. What guiding principles should prevail as the nephrology community creates integrated methods for delivering population care? The primary aims of integrated care should align with the dual goals of the PPACA-defined ACO, namely high-quality patientcentered care at managed per capita costs. However, managing the ESRD population toward these goals will be complex owing to the nature of the population. A typ- ical single Medicare patients with ESRD presents with more than three comorbid conditions, receives 8-10 medications, carries a daily pill burden of 19 pills, and frequently interacts with dialysis and other health care services throughout a given week [12-14]. Such a complex and chronically ill population may be best served using a disease-specific management organization rather than the general ACO model $[5,6]$. Nonetheless, managing the ESRD population will require a subset of pragmatic initiatives designed to improve coordination of care. Such initiatives may include optimizing patient care through better coordination, communication, and care transitions between sites and service providers; prospectively managing comorbid conditions; improving access to service; and reducing avoidable overuse of services. These initiatives should be designed from the patients' point of view in an effort to improve patient experience and satisfaction, ultimately leading to a better quality of life [15].

It is not enough that the nephrology community create and implement initiatives for integrated delivery of renal care. To align with the aims of quality care and cost saving, integration initiatives must be designed to be measured and evaluated. In this way, each initiative provides 
for iterative improvements of the initiative. First, integration initiatives must be designed for accurate and consistent data collection. Providers at every level of the care team must agree to collect pertinent prespecified data, including standard laboratory, process, or outcomes measures commonly used in the discipline. New nonstandard measures may also be identified and defined. For example, providers may be called upon to standardize methods of counting clinical events and conditions, to define new performance or clinical metrics, or to adapt current metrics for innovative analyses. This type of data collection will be supported by emerging electronic health record (EHR) technology and data registry collections. Second, integration initiatives must be designed to track patient outcomes and assess efficacy and efficiency of the initiative. Such efforts will require new collaborations among providers, information technologists, and biostatisticians. To this end, databases must be designed to accommodate large interconnected data sets that include clinical, performance, and financial data. Such databases will support predefined analyses designed to assess clinical quality, patient safety, and cost-effectiveness while controlling for bias or confounding information. To link and analyze clinical performance and financial data, a high level of statistical expertise and methods will be required. It will be through the combined efforts of collecting the right data and analyzing them properly that providers can assess whether integrated delivery of renal care is meeting the expected performance improvement goals.

There is no singularly correct method for a large or small dialysis or physician organization to design and implement a clinical integration initiative that would improve quality of care at reduced costs. In fact, various approaches to similar integration and care problems will probably spark innovation within the current system of largely uncoordinated care. Integration of care is necessary to increase coordination, and there are certainly various approaches to integration. The following intervention examples demonstrate how two large dialysis organizations designed and implemented integration initiatives to address immunization outcomes and oral nutritional supplementation, respectively, in dialysis patients. These examples illustrate the key principles behind improving outcomes in an ACO where the key operating principle is integrated care management: identification of outcomes most in need of improvement in order to affect mortality, hospitalizations, and quality of life; development of robust approaches to improving these outcomes on the basis of current best evidence and best practices; diffusion of these quality approaches throughout the relevant population; measurement and analysis of the effect of each of the approaches; and continuous improvement. All these integration efforts are done in the context of an organization, the ACO, where all the participants, patients, dialysis centers, hospitals, nephrologists, vascular surgeons, and others work together to define the key areas of focus, implement the quality programs, and share in any savings generated (in the case of providers) and in the improved health (in the case of patients).

One large dialysis organization designed and implemented a 3-year integrated effort to increase vaccination rates (i.e., pneumococcal and seasonal influenza vaccination) among dialysis patients, thereby seeking to reduce the risk of hospitalization and death in this population [5]. The integration effort required coordination among facility patient care teams, nephrologists, primary care physicians, operational leadership, and information technology in order to execute a comprehensive system of immunization integration. Specifically, the dialysis organization publicized clear goals, made vaccine available, created automatic data collection and reporting, applied a communications plan, developed standardized tools, and created a culture of patient and team immunization. As a result, vaccination rates in more than 120,000 dialysis patients improved progressively during the integration effort, exceeding the prespecified $90 \%$ immunization rate by the end of the intervention period. These results demonstrate that integration initiatives may reduce healthcare resource utilization for influenza and pneumonia in the dialysis population.

The second large dialysis organization implemented a year-long oral nutritional supplementation program in dialysis patients [6]. Specifically, in an integrated effort among dietitians, clinic staff, nephrologists, and biostatisticians, administration of oral nutritional supplements was monitored in the clinic during dialysis sessions and analyzed in a retrospective study. This initiative reduced the burden on patients and home caregivers to acquire nutritional supplements and track home administration; simultaneously, the initiative provided for increased patient education and encouragement from the clinic staff. As a result, maintenance dialysis patients with albumin concentrations $\leq 3.5 \mathrm{~g} / \mathrm{dl}$ and receiving oral nutritional supplementation demonstrated better survival than matched control patients.

Health care organizations across the nation are already using a disciplined approach to implement and test initiatives toward integrated delivery of care. Most integration efforts share three key elements: performance-based pay- 
ment models, coordination of communication via health care information technology, and developing best practices for care coordination and resource utilization.

Performance-based payment models approximate the proposed ACO model in two ways. First, fee-for-service payments are replaced with prospectively fixed payments, so-called capitated payments, which allow the provider to coordinate the needs for one patient in the context of the entire population while reducing medical costs for the system and securing shared savings for the provider. For example, the physician-led Geisinger Health System is a not-for-profit integrated-delivery health care system serving approximately 2.6 million people in central and northeastern Pennsylvania [16, 17]. Geisinger's product, ProvenCare, charges a fixed rate for services that include preoperative care, surgery, and 90 days of postoperative follow-up care. For coronary artery bypass graft surgery, ProvenCare identifies and groups 40 evidence-based practices that are built into the EHR system as linked sets of orders and reminder prompts. In addition to improved clinical outcomes, improved financial outcomes for ProvenCare coronary artery bypass grafting include a $16 \%$ decrease in average hospital length of stay and a 5\% decrease in hospital costs [18].

The performance-based payment model also approximates the proposed ACO model in that cost savings are shared with the provider in the form of financial incentives available to those who meet prespecified performance goals. Often, base compensation is linked to productivity, and as much as $15-20 \%$ of total compensation is linked to performance, including achieving budget metrics, quality-of-care benchmarks, and patient satisfaction criteria $[18,19]$. Additional stipends and incentives are used to promote skills development and compensate providers for administrative work that is not covered by the fixed payment system but is required to maintain quality performance standards $[18,19]$.

The second key element that is common to integration initiatives is coordination of communication using health care information technology. In fact, information continuity has been identified as a fundamental attribute of an ideal health care delivery system [20], and to that end, numerous health care systems have invested considerable time, effort, and money into developing integrated EHR systems. A recent study of EHR growth in the United States in 2011 reported that nearly $100 \%$ of large integrated health care organizations had adopted EHRs, and 85\% of physicians who had adopted EHRs were somewhat $(47 \%)$ or very (38\%) satisfied [21]. Driven by legislation such as the American Recovery and Reinvestment Act of
2009 and PPACA of 2010, integrated EHR systems are largely but not solely a function of information technology innovation. During development of an integrated EHR system for Kaiser Permanente, clinical leaders provided a frame-work for the structure and total cost of the EHR system; in this way the actual technology was then designed with clinical utility in mind [22]. Such clinical utility was evidenced in the Geisinger Health System upon system-wide deployment of the integrated EHR system, which contained embedded evidence-base guidelines. The number of manually referenced paper medical records decreased by nearly 1 million per year, suggesting that greater access to electronic records across the continuum of care translated to more efficient decision-making in the exam room [18].

In addition to a health care system-wide EHR system, or perhaps because of it, Geisinger and Kaiser Permanente have both successfully deployed online patient portals to enhance communication and provide clinical and financial benefits $[18,22]$. These online portals allow patients to access information, such as benefits packages, laboratory test results, and treatment plans. More important, these online portals provide a mechanism for patients to interact with health care system resources in a way that results in higher physician and clinic productivity. For example, implementation of the MyGeisinger online portal, which included email appointment reminders and electronic access to physicians, was associated with a decreased rate of missed appointments and several thousand fewer telephone calls per month to Geisinger clinics [18].

Finally, common to most integration efforts is a mechanism for cultural change that shifts the providers' focus toward team-based delivery of care. In this model, primary care physicians or nephrologists for patients with ESRD lead a diverse health care team of providers, and outpatient resources are carefully managed to prevent accumulation of unnecessary inpatient expenses. Advocate Physician Partners has demonstrated a clinical integration program that formed the organization around a team culture and was designed to optimize utilization of health care system resources [19]. This program is characterized by several elements. First, Advocate Physician Partners created a single, central leadership team to coordinate integration issues, including outpatient and inpatient care management, transition $\mathrm{s}$ in care management, data collection, and analytics management across the system. Additionally, the Advocate Physician Partners clinical integration program assigned case managers, who were embedded in primary care clinics, to identify complex 
patients and coordinate their services across providers and sites within the health care system. Finally, the clinical integration program increased access to outpatient care: Access to primary care was increased by expanding office hours, after-hours nursing consultations, and collaborations with pharmacy-based clinics, and access to diseasespecific care was increased by creating specialized clinics for patients with poorly controlled chronic conditions.

An ACO-like integrated care model was also deployed by Geisinger Health System in an effort to more aggressively manage outpatient care using a team-oriented approach. In the 2-year pilot project for this clinical integration program, 3000 Medicare patients at two sites were included, and Geisinger reported increases in electronic communication, adherence to prescription medications, and compliance with evidence-based therapies. In those 2 years, the all-cause hospital admission rate declined $20 \%$, equivalent to $7 \%$ cost savings, whereas other Medicare patients demonstrated no change in admission rates [18]. After the program was expanded, a subset of 15,000 patients at 11 sites demonstrated a 5\% reduction of hospital readmission, providing $4 \%$ cost reduction at those sites. Disease-specific management programs were also included to assist complex patients with chronic conditions, such as asthma, hypertension, and CKD. Diabetes disease management programs resulted in $25 \%$ fewer hospital admissions and 43\% fewer days in the hospital. Taken together, these reductions in diabetes-related hospitalization represented a cost savings of more than $\$ 100.00$ per beneficiary per month [18].

Implementation of a clinical integration initiative may be made easier in systems such as Geisinger, Kaiser, or Advocate Physician Partners compared with the nephrology setting because those other systems mainly employ staff physicians, whereas in the nephrology community, dialysis clinics and nephrologists work in collaboration but are not salaried. Nonetheless, close relationships between the dialysis center and nephrologist will provide for cohesive goals, objectives, and implementation strategies. The collaborative nephrology model will probably continue into the future and may even evolve into a more mixed model of staffed, salaried, and contract employees.

Whether future integrated care for the ESRD population is delivered through a general ACO or a renal-focused ACO, clinical integration initiatives will be tailored to specific needs - a particular patient population within a clinic or health care system of a given size and geographic location. Although all these factors will influence the design and application of integrated delivery of care, integration initiatives should be designed us- ing the principles of continuous quality improvement so that efficacy and efficiency can be assessed and improved. Consistent with the this paradigm, integration initiatives should be designed that follow several steps. First, clinical leaders who design integration initiatives must understand the driving undesirable clinical or financial outcomes before creating targeted programs to improve those outcomes. Perhaps most important, leaders must understand how to best measure the clinical or financial outcomes so that the initiative can be designed to measure its own success in influencing the undesirable outcomes. Next, although initiatives should generally focus on health care resource utilization and financial stewardship, each specific initiative should be grounded in a clearly articulated goal and the tasks designed to achieve that goal. For each initiative, there must be a clear process for implementation, and all provider participants must be trained to understand individual roles. Finally, initiatives that were designed to be measured must be analyzed, allowing that the next iteration of the integration initiative may be tailored to better achieving the primary goal. By engaging this continuous quality improvement process, clinical leaders commit to designing initiatives for analysis and improvement.

The nephrology community has a unique opportunity to participate in the restructuring of health care reform in the United States, influencing how disease-specific care is integrated and delivered to the ESRD population. In a shift toward population management, health care organizations will manage populations of patients while individual providers within the organizations will manage individual patients. The general shift toward population management will require a cultural shift that refocuses providers on team-based patient care. Additionally, dedicated organization-wide clinical integration programs must be created to manage the medical, socioeconomic, and practical barriers that prevent providers from delivering integrated health care and prevent patients from participating in an integrated system. Within each clinical integration program, data capture and population analysis will be critical to measuring and quantifying the efficacy and efficiency of each integration effort, and in this regard, information technology innovations will be key to collecting, managing, and analyzing large databases of clinical and financial data. To achieve the dual goals of high-quality care and reduced costs, integration of health care delivery must include commitments from providers at all levels to apply evidence-based care while simultaneously measuring outcomes toward the effort of improving integrated delivery of care. 


\section{Acknowledgments}

We acknowledge Karen Spach and Christopher A. Graybill of DaVita Clinical Research for editorial contribution to the manuscript.

\section{Disclosure Statement}

F.W.M. is an employee of and owns stock in Fresenius Medical Care. S.M. and A.R.N. are employees of and own stocks in DaVita Inc.

\section{References}

1 H.R. 3590, Affordable Care Act. March 22, 2010.

2 National Federation of Independent Business v. Sebelius. 567 U.S._, 132 S.Ct. 2566, 2012 WL 2427810

3 McClellan M, McKethan AN, Lewis JL, Roski J, Fisher ES: A national strategy to put accountable care into practice. Health Aff (Millwood) 2010;29:982-990.

4 Tollen LA: Physician Organization in Relation to Quality and Efficiency of Care: A Synthesis of Recent Literature, New York, NY, The Commonwealth Fund., April 2008.

5 Rizkalla S, Mitchener S, Johnson BJ, van Wyck D, Krishnan M, Nissenson A: Effect of an integrated care delivery system on improving immunization outcomes [Abstract]. J Am Soc Nephrol 2011;22:734A.

6 Lacson E Jr, Wang W, Zebrowski B, Wingard R, Hakim RM: Outcomes associated with intradialytic oral nutritional supplements in patients undergoing maintenance hemodialysis: A quality improvement report. Am J Kidney Dis 2012;60:591-600.

7 Centers for Medicare \& Medicaid Services. [CMS 5003-N] RIN 0938-ZA39 Medicare Program. Demonstration: End-Stage Renal Disease-Disease Management. Fed Regis 2003;68:33495-33506.

8 Arbor Research Collaborative for Health: End-stage renal disease (ESRD) disease management demonstration evaluation report: Findings from 2006-2008, the first three years of a five-year demonstration. Prepared for The Centers for Medicare \& Medicaid Services. 2010. Available at: http://www.cms.gov/ Research-Statistics-Data-and-Systems/Statistics-Trends-and-Reports/Reports/downloads/Arbor_ESRD_EvalReport_2010.pdf. Acccessed March 2012.
9 Nissenson AR, Deeb T, Franco E, Krishnan M, McMurray S, Mayne TJ: The ESRD Demonstration Project: What it accomplished. DaVita Inc. Nephrol News Issues 2011;25: 39-41.

10 Sauer PF, Farrell RE, Lazarus JM: The ESRD demonstration project: What it accomplished. Fresenius Medical Care North America. Nephrol News Issues 2011;25:32, 34-35, 37-38.

11 Centers for Medicare \& Medicaid Services (CMS), HHS: Medicare program; end-stage renal disease prospective payment system. Final rule. Fed Regist 2010;75:49029-49214.

12 Mayne TJ, Burgess M, Weldon J: Finding the case mix adjusters in the CMS proposed bundle. Nephrol News Issues 2009;10:34-38.

13 Chiu YW, Teitelbaum I, Misra M, de Leon EM, Adzize T, Mehrotra R: Pill burden, adherence, hyperphosphatemia, and quality of life in maintenance dialysis patients. Clin J Am Soc Nephrol 2009;4:1089-1096.

14 Manley HJ, Cannella CA, Bailie GR, St Peter WL: Medication-related problems in ambulatory hemodialysis patients: A pooled analysis. Am J Kidney Dis 2005;46:669-680.

15 Strickdorn M, Schneider JS: This Is Service Design Thinking, Hoboken, NJ, John Wiley \& Sons, Inc., 2011.

16 Paulus RA, Davis K, Steele GD: Continuous innovation in health care: Implications of the Geisinger experience. Health Aff (Millwood) 2008;27:1235-1245.
17 Steele GD: Reforming the Healthcare Delivery System. Presented to Committee on Finance United States Senate. 2009. Available at: http://www.finance.senate.gov/imo/media/doc/042109gstest.pdf. Accessed March 2012.

18 McCarthy D, Mueller K, Wrenn J: Geisinger Health System: achieving the potential of system integration through innovation, leadership, measurement, and incentives. The Commonwealth Fund. 2009. Available at: http://www.commonwealthfund.org/Publications/Case-Studies/2009/Jun/GeisingerHealth-System-Achieving-the-Potential-ofSystem-Integration.aspx. Accessed March 2012.

19 Advocate Physician Partners: The 2012 Value Report: Benefits from clinical integration. 2012. Available at: http://www.advocatehealth.com/2012valuereport. Accessed March 2012.

20 Shih T, Davis K, Schoenbaum SC, Gauthier A, Nauzum R, McCarthy D: Organizing the US health care delivery system for high performance. The Commonwealth Fund. 2008. Available at: http://www.commonwealthfund.org/Publications/Fund-Reports/2008/ Aug/Organizing-the-U-S-Health-Care-Delivery-System-for-High-Performance.aspx. Accessed March 2012.

21 Jamoom E, Beatty P, Bercovitz A, Woodwell D, Palso K, Rechtsteiner E: Physician Adoption of Electronic Health Record Systems: United States, 2011. NCHS data brief, no. 98, Hyattsville, MD, National Center for Health Statistics, 2012.

22 Molpus J: Building ACO Foundations: Lessons From Kaiser Permanente's Integrated Delivery Model. Rounds, Danvers, MA, Healthleaders Media, 2012. 